кандидат социологических наук, доцент кафедры менеджмента в отраслях топливно-энергетического комплекса

Тюменского индустриального университета

\section{СТРАТЕГИЯ ВСЕМИРНОЙ ОРГАНИЗАЦИИ ЗДРАВООХРАНЕНИЯ И СОЦИАЛЬНЫЕ ФАКТОРЫ В ПРОФИЛАКТИКЕ РАСПРОСТРАНЕННЫХ НЕИНФЕКЦИОННЫХ БОЛЕЗНЕЙ, ОБУСЛОВЛЕННЫХ ПИТАНИЕМ НАСЕЛЕНИЯ}

\section{Аннотация:}

Уровень и качество жизни современного человека тесно связаны с состоянием его здоровья, на которое оказывают влияние особенности окружающей среды, пищевого поведения самого человека, его двигательная активность и образ жизни. Среди обиирного числа заболеваний особую группу составляют так называемые неинфекционные заболевания, возникновение и развитие которых напрямую связаны с особенностями питания человека. Среди них наиболее известны и распространены сердечно-сосудистые и йододефицитные заболевания. Йододефицитные заболевания развиваются незаметно, но при всей легкости профилактики имеют тяжелые последствия в случае развития, в том числе значительно влияют на интеллект и репродуктивную сферу. В мировой практике в качестве средства профилактики используется йодированная соль, традиционно употребляемая в питании. В то же время избыточное потребление натрия (соли) приводит к развитию сердечно-сосудистых заболеваний, являющихся одной из основных причин смертности в мире. В статье обсуждаются стратегия и рекомендации Всемирной организации здравоохранения по использованию средства профилактики йодного дефицита - йодированной соли, ее влияние на сердечно-сосудистые заболевания $u$ особенности использования. Приводится часть результатов авторского социологического исследования особенностей питания населения и социальных факторов профилактики йододефицитных заболеваний, проведенного в 2013 и 2017 ге. среди учащихся 5-11-х классов школ Тюменской облаcmu $(n=744)$ u ux родителей $(n=677)$.

Ключевые слова:

неинфекционные заболевания, профилактика йододефицитных заболеваний, йодированная соль, Всемирная организация здравоохранения, социологическое исследование, школьники, родители.
PhD in Social Science, Associate Professor, Fuel Energy Sector Management Department, Industrial University of Tyumen

\section{WORLD HEALTH ORGANIZATION STRATEGY AND SOCIAL \\ FACTORS IN THE PREVENTION OF COMMON NONCOMMUNICABLE DISEASES CAUSED BY POPULATION NUTRITION}

\begin{abstract}
Summary:
The level and quality of life of a modern person are closely related to the state of health influenced by environmental risks and eating behavior, physical activity, and lifestyle of a person. The so-called noncommunicable diseases directly related to people's eating habits are considered to be a special group of diseases. Cardiovascular diseases and iodine deficiency disorders are the most wellknown and common noncommunicable diseases. lodine deficiency disorders have no symptoms. However, despite the fact that it is easy to prevent them, they have severe consequences and a great negative effect on intelligence and reproductive sphere. lodized salt, a traditional part of a diet, is a preventive measure in international practice. At the same time, the excessive consumption of sodium (salt) leads to cardiovascular diseases that are a major cause of death in the world. The study discusses the strategy and recommendations of the World Health Organization to use iodized salt to prevent iodine deficiency, reviews its effect on cardiovascular diseases and the aspects of its use. The author presents a fragment of the results of a sociological study on the characteristics of population nutrition and social factors in the prevention of iodine deficiency disorders conducted in 2013 and 2017. The sample included 5th-11th grade students of Tyumen region $(n=744)$ and their parents (n=677).
\end{abstract}

Keywords: noncommunicable diseases, iodine deficiency disorder prevention, iodized salt, World Health Organization, sociological study, school students, parents.

В современном обществе вопросы сохранения здоровья и профилактики заболеваний приобретают все большую актуальность, что обусловлено зависимостью уровня и качества жизни человека от состояния его здоровья. В социологической науке изучению социальных особенностей сохранения здоровья посвящены исследования И.В. Журавлевой, М.А. Захарова, Ф. Зимбардо, Н.А. Лебедевой-Несеври, А.Н. Силина, Е.В. Чернышковой и др. Вопросы здоровья с медико-социальных позиций раскрываются в трудах Ю.П. Лисицына, В.А. Медика, А.М. Осипова, 
А.В. Решетникова, В.К. Юрьева и др. Анализ социальных особенностей питания, пищевых традиций и поведения произведен в работах Д.В. Голоуховой, Н.Н. Зарубиной, С.А. Кравченко, А.В. Носковой и других российских и зарубежных социологов.

Среди обширного числа заболеваний особую группу занимают так называемые неинфекционные заболевания (НИЗ), возникновение и развитие которых напрямую связано с поведенческими фракторами (в первую очередь питанием, двигательной активностью, гигиеной и др.). Среди неинфекционных заболеваний наиболее известны и распространены сердечно-сосудистые и йододефицитные. По данным Всемирной организации здравоохранения (ВО3), в мире ежегодно от НИЗ умирает 41 млн человек (71 \% всех случаев смерти). Более 85 \% «преждевременных смертей» (в возрасте 30-69 лет) приходится на долю стран с низким и средним уровнем доходов. Этим обусловлена постановка цели ВО3: к 2030 г. сократить на одну треть преждевременную смертность от НИЗ посредством профилактической работы [1].

Йододефицитные заболевания (ЙДЗ) определяются ВОЗ как спектр патологических состояний, развивающихся у человека вследствие йодного дефицита (ЙД), возникновение которых можно предотвратить при условии адекватного потребления йода [2]. При этом риск развития ЙДЗ отмечается у 2 млрд жителей планеты, у 740 млн человек диагностирован зоб, а 43 млн имеют умственную отсталость разной степени вследствие ЙД [3]. Природный дефрицит йода наблюдается также на большинстве (около 70 \%) территорий Российской Федерации [4]. Всемирная организация здравоохранения к спектру йододефицитной патологии относит такие заболевания и состояния, как зоб и его осложнения, йод-индуцированный тиреотоксикоз, гипотиреоз, эндемический кретинизм (умственная отсталость, глухонемота, косоглазие, гипотиреоз, карликовость), мертворождение, нарушения умственного и физического развития, когнитивных функций (в том числе нарушения речи, девиантные формы поведения, трудности социальной адаптации).

Наиболее сложной задачей профилактики ЙД является обеспечение ежедневного поступления необходимого количества йода в организм человека с питанием. Для этого в мировой практике используется массовая профилактика, охватывающая все население и обеспечивающая суточный уровень потребления йода (150-200 мкг). Средством профилактики выбрана пищевая соль, обогащенная йодом в соответствии с национальным стандартом. С 1990 по 2017 г. число стран, имеющих дефицит йода в питании населения, сократилось с 113 до 20. К началу 2019 г. в ряде стран, не принявших Закон о всеобщем йодировании соли (ЙС), вопрос о йодном дефиците остается актуальным [5].

Соль является универсальным продуктом для массовой профрилактики ЙДЗ. В числе преимуществ йодированной соли можно назвать следующие.

- Соль потребляется практически всем людьми примерно в одинаковом количестве в течение всего года.

- Это дешевый продукт, который доступен всем слоям населения.

- Исключается вероятность передозировки.

- Не существует противопоказаний для потребления йодированной соли.

- Технологий йодирования соли проста и легко реализуема на любом производстве.

- Йодированная соль в обработанных пищевых продуктах не влияет на их цвет, вкус или запах.

- Качество йодированной соли можно легко контролировать на уровне производства, поставок, торговли и потребления [6].

Расширение производства переработки продуктов питания, быстрая урбанизация и изменение образа жизни изменили модели питания населения. Увеличивается количество и доступность продуктов с высокой степенью переработки. Значительная доля соли поступает в организм человека из обработанных продуктов или при потреблении большого количества хлебобулочных изделий. Соль также добавляется в пищу во время приготовления (бульон и кубики) или за столом (соевый, рыбный соус и поваренная соль). Глутамат натрия используется в качестве пищевой добавки во многих странах мира. Тем не менее основными фракторами, влияющими на потребление натрия, остаются культурный контекст и диетические привычки населения.

По оценкам Федеральной службы по надзору в сфере защиты прав потребителей и благополучия человека, в 42 субъектах РФ здоровье 83,5 млн человек подвергается воздействию рисков отклонения от норм питания [7]. В целях изучения социальных факторов профилактики ЙДЗ и особенностей питания автором в 2013 г. проведено анкетирование учащихся 5-11-х классов школ Тюменской области $(\mathrm{n}=744)$ и их родителей $(\mathrm{n}=677)$ (гнездовая выборка, статистическая погрешность - 4,0 \% при $p=0,95)$. В 2017 г. (совместно с О.Б. Макаровой, Л.А. Суплотовой, Г.В. Шарухо) опрос проведен повторно.

Согласно результатам исследования, в понимании большинства школьников питаться правильно - значит: не злоупотреблять жирной, острой пищей $(59,7 \%)$, не есть фрастфуд $(57,7)$, не переедать $(57,0)$, есть фррукты каждый день $(52,8)$, не есть много сладкого $(51,9 \%)$. Большая часть 
родителей отмечает, что нельзя злоупотреблять жирной, острой пищей $(55,7 \%)$, есть фрастфуд $(56,4)$, нужно есть в меру $(59,2)$ и регулярно $(53,0)$, отдавать предпочтение домашней пище $(55,2 \%)$.

Наблюдая за распределением ответов школьников в зависимости от ответов их родителей на вопрос: «Часто ли твоя мама говорит: "Мы следим за питанием, надо есть полезные продукты"?», можно отметить следующее. При любых ответах родителей подавляющее большинство школьников (43-56 \%) отмечает пункт «иногда»; чем более интенсивно влияние мнения родителей, тем чаще замечают его дети ( $\chi^{2}$ Пирсона $=32,48, d f=15, p=0,006 ; V$ Крамера $=0,130$; $\mathrm{n}=645)$. Самые обсуждаемые темы о питании: вред сладких напитков $(54,0 \%$ школьников), полезные продукты $(38,4)$, вред фрастфуда $(41,4)$, вредные для здоровья продукты $(37,9)$, вред чрезмерного употребления сладкого $(47,3 \%)$.

В 2013 г. более половины родителей $(53,2$ \%) заметили, что йодированную соль в домашнем питании использовали ежедневно, 8,7 \% не использовали, 10,1 \% затруднились с ответом, 28,0 \% никогда использовали ЙС в питании. В 2016-2017 гг. был разработан и осуществлен план мероприятий «Реализация нового информационного (просветительского) проекта по профилактике йодного дефицита в Тюменской области». По результатам мониторинга, проведенного в 2017 г., можно отметить, что на 12,0 \% увеличилось число родителей, отслеживающих ежедневное потребление ЙС в домашнем питании. Так, выбирая соль в магазине, 37,1 \% купят йодированную, 31,3 - любую, 7,4 - морскую, 6,8 - нейодированную, 17,4 \% респондентов затруднились с ответом. При этом половина опрошенных (49,2 \%) отметила, что определяющим фактором для них является российский производитель ИС.

22 сентября 2016 г. ВОЗ в рамках Генеральной Ассамблеи ООН анонсировала проект Global Hearts. Инициатива направлена на профилактику сердечно-сосудистых заболеваний, включая сердечные приступы и инсульты, как ведущей в мире причины смертности. Предлагается принятие мер по профилактике сердечно-сосудистых заболеваний, включая введение налога на табак, сокращение соли в продуктах питания, выявление и лечение людей с высоким риском развития заболеваний и улучшение качества первичной медицинской помощи. Используя такие меры, в США удалось сократить смертность от сердечно-сосудистых заболеваний более чем на $40 \%$, а в Финляндии показатели смертности от подобных заболеваний среди мужчин снижены на 80 \% [8]. Предлагаемые мероприятия целесообразно проводить в России, учитывая актуальность сердечно-сосудистых заболеваний и вызываемую ими высокую смертность.

Таким образом, низкое потребление йодированной соли приводит к развитию ЙДЗ, а избыточное - сердечно-сосудистых заболеваний. В связи с этим государства - члены ВОЗ в 2013 г. (резолюция WHA 66.10) сформулировали глобальную цель профрилактики неинфекционных заболеваний 30 \% относительного сокращения среднего потребления соли с целью достижения к 2025 г. целевого показателя менее 5 г в день (приблизительно 2 г натрия) [9]. При этом вся потребляемая соль должна быть йодированная. Вторая договоренность -добиться к 2025 г. 25 \%-го снижения распространенности повышенного кровяного давления. Достижение поставленных целей, по оценкам ВОЗ, позволит ежегодно предотвратить около 2,5 млн смертей [10].

ВОЗ выделила три аспекта стратегии по сокращению потребления натрия: создание условий, позволяющих снизить потребление натрия, и просвещение потребителей, чтобы побудить их сделать выбор в пользу снижения содержания натрия в рационе; оценка и мониторинг потребления натрия; анализ эффективности стратегии, направленной на сокращение потребления соли и восполнение недостатка йода [11].

Для профилактики ЙДЗ и сокращения потребления соли необходимы:

- политика правительства, которая должна включать соответствующие налогово-бюджетные меры и регулирование, чтобы производители были заинтересованы в изготовлении более здоровой пищи, а розничные продавцы делали ее доступной для населения; обеспечение обязательного йодирования пищевой поваренной соли и контроль ее качества;

- организация информирования потребителей посредством социального маркетинга с использованием социально дифференцированного подхода для повышения осведомленности всех слоев населения;

- мониторинг потребления соли, просвещение населения об источниках соли в рационе, формирование у граждан правильного отношения и поведения по вопросам, связанным с солью.

Таким образом, для достижения эффективности профилактики необходима целенаправленная работа по снижению воздействия фракторов риска развития болезней. В целях сокращения негативного воздействия неинфекционных заболеваний на население требуется комплексный подход, вовлекающий в профилактическую работу различные институты общества. Реализуемые программы профилактики дефицита йода и сокращения употребления натрия должны обеспечивать населению доступ к безопасным продуктам питания, включая соль с пониженным содержанием натрия и достаточным содержанием йода. 


\section{Ссылки:}

1. Неинфекционные заболевания [Электронный ресурс] // Всемирная организация здравоохранения. 2018. 1 июня. URL: http://www.who.int/mediacentre/factsheets/fs355/ru/ (дата обращения: 05.06.2019).

2. Цит. по: Йододефицитные заболевания в Российской Федерации: время принятия решений / И.И. Дедов, Г.А. Мельниченко, Е.А. Трошина, Н.М. Платонова, Ф.М. Абдулхабирова, Г.А. Герасимов. М., 2012. 232 с.

3. Iodine Deficiency in 2007: Global Progress Since 2003 / B. de Benoist, E. McLean, M. Andersson, L. Rogers // Food and Nutrition Bulletin. 2008. Vol. 29, no. 3. P. 195-202. https://doi.org/10.1177/156482650802900305.

4. Йододефицитные заболевания ...

5. lodine Deficiency in $2007 \ldots$

6. Федак И.Р. Контрольно-эпидемиологические исследования эфффективности региональных программ профилактики йододефицитных заболеваний в РФ : автореф. дис. ... канд. мед. наук. М., 2008. 30 с.

7. О состоянии санитарно-эпидемиологического благополучия населения в Российской Федерации в 2017 г. : государственный доклад. М., 2018. 268 с.

8. Global Hearts Initiative, Working Together to Promote Cardiovascular Health [Электронный ресурс] // World Health Organization. 2016. URL: https://www.who.int/cardiovascular diseases/global-hearts/en/ (дата обращения: 05.06.2019).

9. Salt Reduction and lodine Fortification Strategies in Public Health [Электронный ресурс] : Report of a Joint Technical Meeting Convened by World Health Organization and the George Institute for Global Health in Collaboration with the International Council for the Control of lodine Deficiency Disorders. Sydney, 2014. 36 p. URL: https://apps.who.int/iris/bitstream/handle/10665/101509/9789241506694 eng.pdf;jsessionid=89C1F9D5213033D750B9DBF4C37036C3?sequence=1 (дата обращения: 05.06.2019).

10. Ibid.

11. Ibid.

\section{References:}

Benoist, B de, McLean, E, Andersson, M \& Rogers, L 2008, 'lodine Deficiency in 2007: Global Progress Since 2003', Food and Nutrition Bulletin, vol. 29, no. 3, pp. 195-202, https://doi.org/10.1177/156482650802900305.

Global Hearts Initiative, Working Together to Promote Cardiovascular Health 2016, World Health Organization, viewed 05 June 2019, <https://www.who.int/cardiovascular diseases/global-hearts/en/>

Fedak, IR 2008, Control and Epidemiological Studies of the Effectiveness of Regional Programs for the Prevention of lodine Deficiency Diseases in Russia, PhD thesis abstract, Moscow, 30 p., (in Russian).

Noncommunicable Diseases 2018, World Health Organization, June 1, viewed 05 June 2019, <http://www.who.int/mediacentre/factsheets/fs355/ru/>.

On the State of Sanitary and Epidemiological Welfare of the Population in the Russian Federation in 2017: State Report 2018, Moscow, 268 p., (in Russian).

Salt Reduction and lodine Fortification Strategies in Public Health: Report of a Joint Technical Meeting Convened by World Health Organization and the George Institute for Global Health in Collaboration with the International Council for the Control of lodine Deficiency Disorders 2014, Sydney, 36 p., viewed 05 June 2019, <https://apps.who.int/iris/bitstream/handle/10665/101509/9789241506694 eng.pdf;jsessionid=89C1F9D5213033D750B9DBF4C37036C3?sequence=1>

Troshina, EA, Platonova, NM, Abdulhabirova, FM, Gerasimov, GA, Dedova, II \& Melnichenko, GA (eds) 2012, lodine Deficiency Disorders in the Russian Federation: the Time of Decision-making, Moscow, 232 p., (in Russian). 